\title{
Risk of tuberculosis in patients with solid cancers and haematological malignancies: a systematic review and meta-analysis
}

\author{
Claudia C. Dobler (10 ${ }^{1,2,3}$, Kelvin Cheung ${ }^{1,2}$, John Nguyen ${ }^{1,2}$ and Andrew Martin ${ }^{4}$ \\ Affiliations: 'Department of Respiratory Medicine, Liverpool Hospital, Liverpool (Sydney). NSW, Australia. \\ ${ }^{2}$ South Western Sydney Clinical School, University of New South Wales, Sydney, NSW, Australia. ${ }^{3}$ Clinical \\ Management Group, Woolcock Institute of Medical Research, University of Sydney, NSW, Australia. ${ }^{4}$ NHMRC \\ Clinical Trials Centre, University of Sydney, NSW, Australia.
}

Correspondence: Claudia C. Dobler, Department of Respiratory Medicine, Liverpool Hospital, Elizabeth Street, Liverpool, NSW 2170, Australia. E-mail: c.dobler@unsw.edu.au

@ERSpublications

Screening and treatment for latent tuberculosis infection should be considered in children with cancer http://ow.ly/lpSQ30dxyam

Cite this article as: Dobler CC, Cheung K, Nguyen J, et al. Risk of tuberculosis in patients with solid cancers and haematological malignancies: a systematic review and meta-analysis. Eur Respir J 2017; 50: 1700157 [https://doi.org/10.1183/13993003.00157-2017].

ABSTRACT There is uncertainty regarding whether patients with cancer should be screened for latent tuberculosis infection (LTBI). We performed a systematic review and meta-analysis to estimate the relative incidence of tuberculosis $(\mathrm{TB})$ in cancer.

We searched MEDLINE and Embase for studies published before December 21, 2016. We included studies that evaluated the incidence of TB in patients with solid cancers and haematological malignancies relative to a reference group (study control or general population). A pooled estimate of the incidence rate ratio (IRR) was obtained using standard meta-analysis methods.

The search strategy identified 13 unique studies including 921464 patients with cancer. The IRR of TB for adult patients with cancer was 2.61 (95\% CI 2.12-3.22; $\left.I^{2}=91 \%\right)$. In haematological cancers, the IRR was 3.53 (95\% CI $1.63-7.64 ; I^{2}=96 \%$ ); and in solid cancers in adults, it was 2.25 (95\% CI 1.96-2.58; $\left.I^{2}=91 \%\right)$. The highest IRR was found in children with haematological malignancies or solid cancers (IRR $16.82,95 \%$ CI $\left.8.81-32.12 ; I^{2}=79 \%\right)$.

Considering the limited duration of maximum immunosuppression in cancer and reduced cumulative lifetime risk of TB because of reduced life expectancy, children, but not adults, appear to be at a sufficient level of risk to warrant systematic screening for LTBI.

This article has supplementary material available from erj.ersjournals.com

Received: Jan 232017 | Accepted after revision: May 262017

Support statement: C.C. Dobler was supported by an Australian National Health and Medical Research Council fellowship (APP1090198). The funder had no role in study design, data collection, data analysis, data interpretation, or writing of the manuscript. The corresponding author had full access to all data in the study and had final responsibility for the decision to submit for publication. Funding information for this article has been deposited with the Crossref Funder Registry.

Conflict of interest: None declared

Copyright @ERS 2017 


\section{Introduction}

Tuberculosis (TB) is one of the major infectious causes of disease and death globally [1]. There is currently a renewed interest in screening and treatment of latent tuberculosis infection (LTBI), as a possible means to achieve control of the global tuberculosis epidemic [2]. In most infected people, TB remains clinically asymptomatic and microbiologically inactive (latent). However, in approximately 5 to $10 \%$ of latently infected persons, the infection will cause active TB at some point during their lifetime [3]. The risk of TB reactivation is increased in persons with immunocompromising conditions, such as HIV infection [4], chronic renal failure [5] or diabetes mellitus [6], and in persons on immunocompromising medications, such as tumour necrosis factor- $\alpha$ (TNF- $\alpha$ ) inhibitors $[7,8]$. The World Health Organization (WHO) recommends targeted screening of high risk groups in high or upper middle income countries with a TB incidence of $<100$ per 100000 per year [9], as preventive TB treatment can significantly reduce the risk of TB reactivation [10].

Patients with solid cancers and haematological malignancies are immunocompromised because of the disease itself, and as a consequence of chemotherapy. It is therefore reasonable to assume that the risk of $\mathrm{TB}$ reactivation would be increased in people with cancer, and consider LTBI screening and treatment in this group. There is, however, a paucity of information on whether patients with cancer should be screened for LTBI. The British National Institute for Health and Care Excellence (NICE) recommendations state that people who "have a haematological malignancy", "are having chemotherapy" and "have had a gastrectomy" (for gastric cancer or other reasons) are at increased risk of developing TB, but they do not provide any specific screening and treatment recommendations for these groups [11]. Joined guidelines of the American Thoracic Society (ATS) and the Centers for Disease Control and Prevention (CDC), endorsed by the Council of the Infectious Diseases Society of America (IDSA), identify persons with "some hematologic disorders (e.g., leukemias and lymphomas)" and "other specific malignancies (e.g., carcinoma of the head or neck and lung)" as high risk, and recommend the consideration of treatment of LTBI in these groups [12]. The evidence that has informed these guidelines was derived from an informal synthesis of individual studies conducted from the 1950s to 1970s that had important methodological limitations: the frequency of $\mathrm{TB}$ among patients with cancer was expressed as a proportion (cumulative incidence) rather than an incidence rate; thus not adjusting for the observation period/ time at risk. Relative risk estimates were calculated by dividing the cumulative incidence of TB in a cancer cohort by an incidence rate (per year) in the general population [13], or were not calculated at all [14].

A recently published systematic review and meta-analysis by Cheng et al. [15] on the risk of TB in patients with cancer also had significant methodological limitations. That analysis did not exclude studies that contained information on cumulative incidence of TB only, and used annual country-specific TB incidence rates that were unadjusted for potential confounders obtained from WHO for comparison [15]. Comparing the cumulative incidence of TB in patients with cancer (in one included study cumulated over a study period of 25 years [16]) with an annual TB incidence in the general population would have resulted in overestimation of the risk of TB in patients with cancer. Equally, using the general population as a comparator group, without any adjustment for potential confounders, particularly age, would have resulted in overestimation of the risk of TB in patients with cancer. The authors' conclusion of the study that individuals living in the United States with haematologic, head and neck, and lung cancers would benefit from targeted LTBI screening and therapy thus has to be questioned.

Another systematic review published in 2014 focused on lung cancer only, and evaluated the prevalence of TB in those patients [17]. It was not the goal of that review to establish causality (the authors were aware that there is a bidirectional causal link between lung cancer and TB [18]).

There remains considerable uncertainty about whether patients with cancer should be screened for LTBI, with the intention to offer preventive treatment, if there is evidence of LTBI. The risk of TB in cancer patients remains imprecisely quantified, despite the need for contemporary evidence to inform guidelines and public health policy on LTBI screening and treatment in this setting. We have consequently performed a systematic review and meta-analysis to estimate the relative incidence of TB in people with cancer (solid cancers as well as haematological malignancies) in comparison to the general population.

\section{Methods}

\section{Search strategy and selection criteria}

We searched the electronic databases MEDLINE, including Epub Ahead of Print, In-Process \& Other Non-Indexed Citations (1946 to December 21, 2016) and Embase (1947 to December 21, 2016), through OVID for studies that examined the risk of $\mathrm{TB}$ in cancer (solid tumours and haematological malignancies). The search strategy was discussed and agreed upon by the authors, and advice was sought from an experienced research librarian. The following search terms were used to identify citations relevant to (1) tuberculosis, (2) risk and (3) cancer, respectively: (1) tuberculosis, Mycobacterium tuberculosis; (2) 
risk, incidence, probability, proportion, frequency; and (3) neoplasm, cancer, malignancy, tumour, carcinoma, oncology and metastases. The detailed search strategy is available in the supporting information (S1 Appendix). Articles resulting from these searches and relevant references cited in those articles were reviewed. In addition, we screened reference lists of systematic reviews, literature reviews and meta-analyses identified through the search. Articles published in any language were included.

Eligible studies evaluated the incidence of TB in patients with cancer, relative to a reference group (control group specified for the study, or general population with or without adjustment for potential confounding factors), allowing for estimation of the relative risk of TB in cancer. We included studies reporting on pulmonary, as well as extra-pulmonary TB. A diagnosis of TB could either have been based on microbiological results, or symptoms and chest radiographic findings consistent with $\mathrm{TB}$. Studies were included that reported on solid cancers or haematological malignancies in any age group. Studies that reported on the risk of TB in patients who received a stem cell transplant were excluded. We excluded cross-sectional (prevalence) studies, studies that used cumulative incidence (incidence proportion) without adjustment for the time at risk, studies in which a diagnosis of TB preceded the cancer diagnosis and studies in which the temporal relationship between TB and cancer was not specified. Furthermore, we excluded studies that only examined the risk of TB in pre-defined subgroups of cancer patients that were considered to have an increased pre-test probability of TB infection (e.g. because of abnormal chest radiographs). Abstracts, editorials, case reports, conference abstracts, systematic reviews, literature reviews and meta-analyses were not included. Three reviewers (C.C. Dobler, K. Cheung and J. Nguyen) independently screened titles and abstracts and then the full text of papers identified as potentially eligible by at least one reviewer. Disagreements between the reviewers were resolved by discussion. This systematic review follows the PRISMA (Preferred Reporting Items for Systematic Reviews and Meta-Analyses) guidelines for reporting meta-analyses and systematic reviews of observational studies [19].

\section{Data analysis}

Three reviewers (C.C. Dobler, K. Cheung and J. Nguyen) independently extracted data from included studies onto a standardised data sheet. Variables recorded from each article included the following:

Publication year, country, study design, number of patients, incidence of TB in the cancer group, incidence of $\mathrm{TB}$ in the reference group (i.e. a control sample or applicable general population statistic), time of follow-up, demographic and clinical characteristics of patients, previous TB (based on history, chest radiographs, etc.), prevalence of LTBI based on the tuberculin skin test (TST) or interferon gamma release assay (IGRA) and treatment of LTBI, type of cancer, information on chemotherapy and TB definition for the purpose of the study. Risk of bias in the included studies was evaluated using the Newcastle-Ottawa Quality Assessment Scale for Cohort Studies [20].

For studies in which an IRR was not specifically reported, the IRR was calculated by dividing the incidence of TB in cancer patients by the incidence of TB in the reference group. If not reported, a $95 \%$ CI for the IRR was determined, assuming the frequency of observed cases followed a Poisson distribution [21]. We accepted hazard ratios (HRs), the ratio of the hazard (i.e. the "instantaneous" rate) in cancer patients compared to a control group or the general population, as estimates of IRRs [22]. For studies that reported a crude IRR, as well as an adjusted or standardised IRR, we included the adjusted or standardised IRR (aIRR and standardised incidence ratio (SIR), respectively) in the meta-analysis. We used DerSimonian and Laird random effects meta-analyses [23] with the Comprehensive Meta-Analysis v3.0 software (Biostat, Englewood, NJ, USA) to estimate pooled IRRs and 95\% CIs. We assessed the magnitude of heterogeneity using the $I^{2}$ statistic, and tested for its statistical significance using the Q-statistic. Subgroup analysis was pre-specified for age groups (adults versus children), as well as for any specific solid tumours or haematological malignancies.

As there was a maximum of one event (TB diagnosis) per patient or control in the included studies, the IRR expressed the relative risk for TB, and the terms were thus used interchangeably.

\section{Results}

The search strategy identified 11006 unique citations, of which 42 were included for full-text review and 13 studies met the inclusion and exclusion criteria, involving a total of 921464 patients with cancer (figure 1). Table 1 summarises the characteristics of the included studies [24-36].

\section{Study characteristics}

All 13 studies were observational and retrospective. Five studies were based on information from national databases and eight were cohort studies at a single hospital site. Five studies were from Taiwan, five from South Korea, two from South Africa, and one from the USA. Four studies included patients with different haematological and solid cancers; one study each, reported only on the risk of TB in patients with 


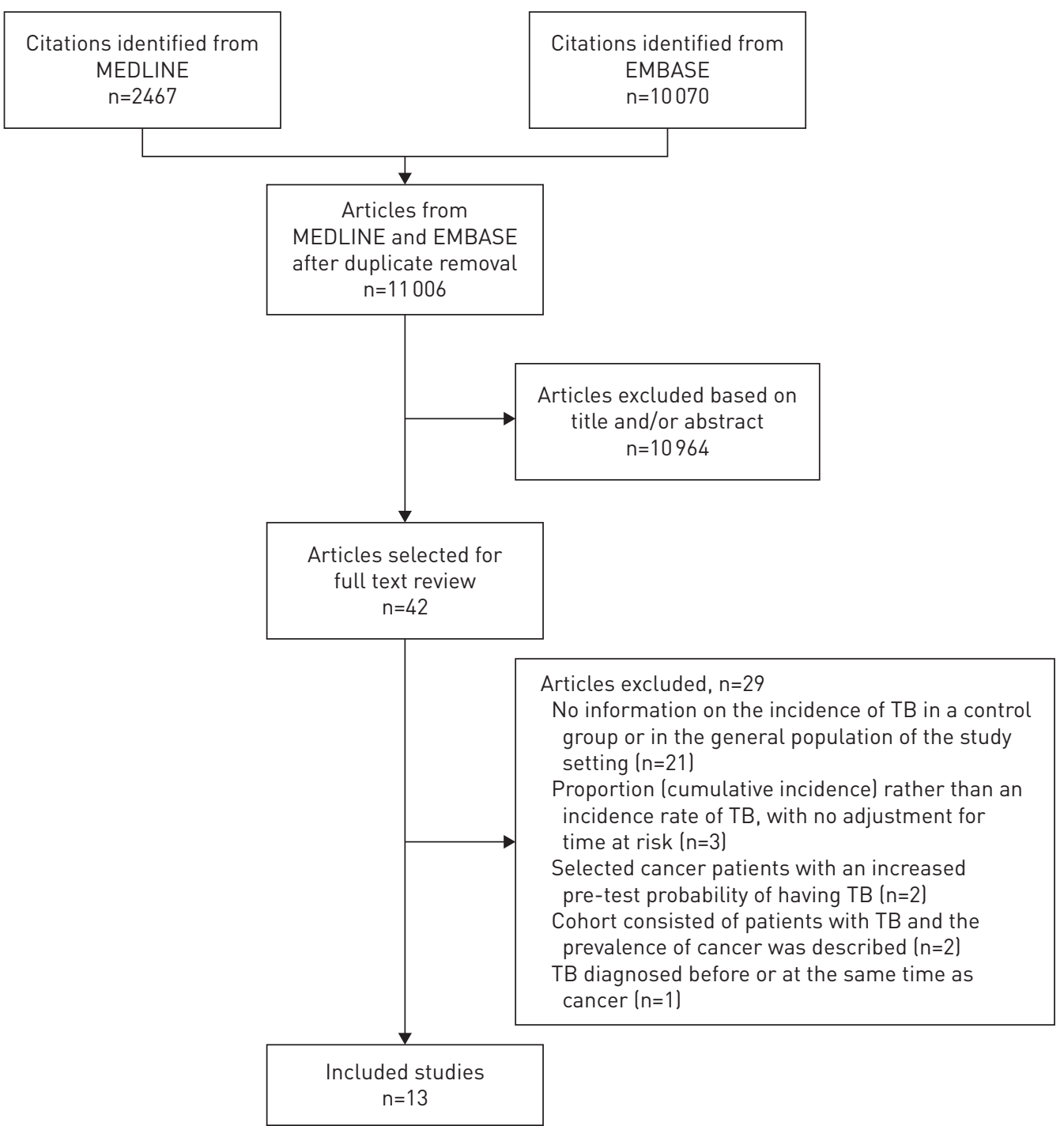

FIGURE 1 Flowchart of study selection.

different haematological or solid cancers, respectively; and seven studies included only patients with a specific cancer type (five studies on gastric cancer, one study on lung cancer and one study on chronic myeloid leukaemia (CML)). Two studies from South Africa focused on children (aged up to 15 years) with different haematological and solid malignancies. Study size ranged from 257 to 855382 participants.

A common reason for the exclusion (from this systematic review) of studies that evaluated the risk of $\mathrm{TB}$ in patients with cancer after a full-text review was the lack of information on the incidence of TB in an appropriate reference group. Furthermore, several studies were excluded because they expressed TB cases among cancer patients as a proportion (cumulative incidence) rather than an incidence rate, with no adjustment for the time at risk $[16,37,38]$. Two studies were excluded after full-text review because they reported the risk of TB in cancer patients with an increased pre-test probability of having TB (based on the assessment of clinicians [39] or chest radiographic abnormalities suspicious for TB [40]). Another study was excluded because TB was diagnosed simultaneously with cancer in the majority of cases [41]. In two excluded studies, the examined cohort consisted of patients with TB, and the prevalence of cancer was described $[42,43]$.

Adjustments for confounding in studies included in the meta-analysis are outlined in table 1 .

\section{Relative risk of TB in patients with cancer}

The overall IRR of TB among adult persons with cancer was 2.61 (95\% CI, 2.12-3.22) (figure 2). There was considerable heterogeneity between studies ( $Q$-statistic, $\mathrm{p}<0.001, I^{2}=91 \%$ ). The pooled IRR of the two 
TABLE 1 Characteristics of included studies

\begin{tabular}{|c|c|c|c|c|c|c|c|c|}
\hline $\begin{array}{l}\text { Study, year of } \\
\text { publication } \\
\text { [ref.] } \\
\text { Study period }\end{array}$ & Population and setting & Cancer type & Cancer treatment & $\begin{array}{c}\text { Control/general } \\
\text { population }\end{array}$ & $\begin{array}{l}\text { TB cases and } \\
\text { incidence in } \\
\text { cancer group }\end{array}$ & $\begin{array}{l}\text { TB cases and } \\
\text { incidence in } \\
\text { control group }\end{array}$ & $\begin{array}{l}\text { Rate } \\
\text { ratio } \\
{[95 \%} \\
\text { CI) }\end{array}$ & $\begin{array}{l}\text { Adjustment for } \\
\text { potential } \\
\text { confounders }\end{array}$ \\
\hline $\begin{array}{c}\text { Wu } 2011[24] \\
2000-2007\end{array}$ & $\begin{array}{c}\mathrm{n}=16487 \text { cancer patients } \\
\text { in Taiwan (no age } \\
\text { restriction) } \\
\text { Taiwan national } \\
\text { population (NHI } \\
\text { database) }\end{array}$ & $\begin{array}{l}\text { All solid organ } \\
\text { cancers and } \\
\text { haematological } \\
\text { malignancies, IRRs } \\
\text { given for cancer } \\
\text { subgroups }\end{array}$ & not specified & $\begin{array}{c}\mathrm{n}=65948 \\
\text { controls from } \\
\text { the NHI } \\
\text { database without } \\
\text { cancer, matched } \\
\text { for age and sex }\end{array}$ & $\begin{array}{l}205 \text { cases out of } \\
16487 \text { patients; no } \\
\text { information on } \\
\text { median follow-up } \\
\text { time } \\
\text { TB incidence: } \\
339 / 100000 \\
\text { person-years }\end{array}$ & $\begin{array}{l}489 \text { cases out } \\
\text { of } 65948 \\
\text { controls } \\
\text { TB incidence: } \\
202 / 100000 \\
\text { person-years }\end{array}$ & $\begin{array}{c}\text { alRR } \\
1.67 \\
(1.42- \\
1.96)\end{array}$ & $\begin{array}{l}\text { controls matched } \\
\text { for age and sex, } \\
\text { and IRR adjusted } \\
\text { for age, sex and } \\
\text { comorbidities in } \\
\text { multivariate } \\
\text { analysis }\end{array}$ \\
\hline $\begin{array}{c}\text { Seo } 2016[25] \\
2008-2012\end{array}$ & $\begin{array}{c}\mathrm{n}=855382 \text { patients with } \\
\text { newly diagnosed cancer } \\
\text { in Korea, aged } 20 \text { to } \\
99 \text { years, identified from } \\
\text { the National Health } \\
\text { Insurance Review } \\
\text { Assessment Service } \\
\text { (HIRA) } \\
\text { excluded: patients with } \\
\text { lung cancer }\end{array}$ & $\begin{array}{c}\text { thyroid cancer } \\
\text { (n=170 597), gastric } \\
\text { cancer ( } n=253 \text { 158), } \\
\text { hepatocellular } \\
\text { carcinoma ( } n=83512 \text { ), } \\
\text { colon cancer } \\
\text { (n=77 903), breast } \\
\text { cancer ( } n=75 \text { 801), } \\
\text { haematological } \\
\text { malignancy } \\
\text { (n=32 026), pancreatic } \\
\text { cancer ( } n=22822 \text { ), } \\
\text { biliary tract cancer } \\
\text { (n=20 452), other } \\
\text { cancers ( } n=219111 \text { ) }\end{array}$ & not specified & $\begin{array}{c}\text { General } \\
\text { population of } \\
\text { Korea aged } 20 \text { to } \\
99 \text { years }\end{array}$ & $\begin{array}{l}5745 \text { cases out of } \\
855382 \text { patients } \\
\text { (1589 } 876 \\
\text { patient-years of } \\
\text { follow-up), median } \\
\text { follow-up time: } 1.6 \\
\text { years } \\
\text { TB incidence } \\
361.3 / 100000 \\
\text { person-years of } \\
\text { follow-up }\end{array}$ & $\begin{array}{c}230247 \text { cases } \\
\text { in the general } \\
\text { population } \\
\text { TB incidence: } \\
125.3 / 100000 \\
\text { person-years }\end{array}$ & $\begin{array}{c}\text { SIR } \\
2.22 \\
(2.17- \\
2.27)\end{array}$ & $\begin{array}{l}\text { TB incidence was } \\
\text { standardised for } \\
\text { age and sex }\end{array}$ \\
\hline $\begin{array}{c}\text { Chen 2011 [26] } \\
\text { 1996-2009 }\end{array}$ & $\begin{array}{c}\mathrm{n}=2984 \text { adult cancer } \\
\text { patients ( }>18 \text { years) } \\
\text { HIV+ excluded } \\
\text { National Taiwan } \\
\text { University Hospital, } \\
\text { Taiwan }\end{array}$ & $\begin{array}{c}\text { haematological } \\
\text { malignancies } \\
\text { AML (n=1011), ALL } \\
(n=276), \text { lymphoma } \\
(n=956), M M(n=307), \\
\text { CML/CLL }(n=169), \\
\text { SAA/MDS ( }=265)\end{array}$ & not specified & $\begin{array}{c}\text { general } \\
\text { population of } \\
\text { Taiwan }\end{array}$ & $\begin{array}{l}53 \text { cases out of } \\
2984 \text { patients; no } \\
\text { information on } \\
\text { median follow-up } \\
\text { time } \\
\text { TB incidence: } \\
120 / 100000 \text { per } \\
\text { year }\end{array}$ & $\begin{array}{l}\text { number of TB } \\
\text { cases and size } \\
\text { of control } \\
\text { population not } \\
\text { given } \\
\text { TB incidence: } \\
62 / 100000 \text { in } \\
2008\end{array}$ & $\begin{array}{l}\text { IRR } \\
1.94^{\#} \\
(1.48- \\
2.53)^{\top 1}\end{array}$ & no adjustment \\
\hline $\begin{array}{r}\text { Liu } 2015 \text { [27] } \\
1998-2011\end{array}$ & $\begin{array}{l}\mathrm{n}=1082 \text { patients with } \\
\text { newly diagnosed } \mathrm{CML} \text {, } \\
\text { aged } 20 \text { years and older, } \\
\text { identified from } \mathrm{NHI} \\
\text { database in Taiwan }\end{array}$ & CML & $\begin{array}{l}4.5 \% \text { had a } \\
\text { haematopoietic stem } \\
\text { cell transplantation, } \\
23.3 \% \text { received } \\
\text { interferon-alpha, all } \\
\text { CML patients } \\
\text { received at least one } \\
\text { type of Bcr-Abl } \\
\text { tyrosine } \\
\text { kinase inhibitor }\end{array}$ & $\begin{array}{l}\text { Subjects without } \\
\text { CML from the } \\
\text { NHI database in } \\
\text { Taiwan, matched } \\
\text { for age, sex and } \\
\text { comorbidities }\end{array}$ & $\begin{array}{l}19 \text { cases out of } \\
1082 \text { patients, } \\
\text { median follow-up } \\
\text { time: } 3.9 \text { years } \\
\text { TB incidence: } \\
401 / 100000 \\
\text { person-years }\end{array}$ & $\begin{array}{l}59 \text { cases out of } \\
10820 \\
\text { matched } \\
\text { subjects in the } \\
\text { control cohort } \\
\text { TB incidence: } \\
105 / 100000 \\
\text { person-years }\end{array}$ & $\begin{array}{c}\text { Crude } \\
\text { HR } 3.65 \\
(2.18- \\
6.12) \\
\text { aHR } \\
3.76 \\
(2.24- \\
6.31)\end{array}$ & $\begin{array}{l}\text { controls matched } \\
\text { by age, sex and } \\
\text { comorbidities, } \\
\text { and HR adjusted } \\
\text { for age, sex and } \\
\text { comorbidities in } \\
\text { multivariate } \\
\text { analysis }\end{array}$ \\
\hline
\end{tabular}




\begin{tabular}{|c|c|c|c|c|c|c|c|c|}
\hline $\begin{array}{l}\text { Study, year of } \\
\text { publication } \\
\text { [ref.] } \\
\text { Study period }\end{array}$ & Population and setting & Cancer type & Cancer treatment & $\begin{array}{l}\text { Control/general } \\
\text { population }\end{array}$ & $\begin{array}{l}\text { TB cases and } \\
\text { incidence in } \\
\text { cancer group }\end{array}$ & $\begin{array}{l}\text { TB cases and } \\
\text { incidence in } \\
\text { control group }\end{array}$ & $\begin{array}{l}\text { Rate } \\
\text { ratio } \\
(95 \% \\
\text { CI) }\end{array}$ & $\begin{array}{l}\text { Adjustment for } \\
\text { potential } \\
\text { confounders }\end{array}$ \\
\hline $\begin{array}{l}\text { Kim 2008 [28] } \\
2001\end{array}$ & $\begin{array}{c}\mathrm{n}=1809 \text { cancer patients } \\
\text { in a tertiary hospital in } \\
\text { South Korea } \\
\text { median age } 58 \text { years } \\
\text { (range, 15-90) } \\
\text { excluded: HIV+, silicosis, } \\
\text { immunosuppressive } \\
\text { therapy other than } \\
\text { corticosteroids for } \\
\text { cancer treatment }\end{array}$ & $\begin{array}{c}\text { gastric cancer } \\
\text { (n=663), } \\
\text { liver cancer ( } n=382), \\
\text { lung cancer( } n=348), \\
\text { colon cancer ( } n=277 \text { ) } \\
\text { and breast cancer } \\
\text { ( } n=139 \text { ) } \\
\text { IRRs can be } \\
\text { calculated for cancer } \\
\text { subgroups based on } \\
\text { information provided }\end{array}$ & $\begin{array}{c}\mathrm{n}=1132(62.6 \%) \text { had } \\
\text { surgery, } \mathrm{n}=757 \\
(41.8 \%) \text { had } \\
\text { chemo therapy, } \\
\text { of gastric cancer } \\
\text { patients, } 459 \text { out of } \\
663(69 \%) \text { had } \\
\text { gastrectomy }\end{array}$ & $\begin{array}{c}\mathrm{n}=1809 \text { controls, } \\
\text { patients } \\
\text { attending the } \\
\text { same hospital } \\
\text { during the same } \\
\text { time period for } \\
\text { the } \\
\text { treatment of } \\
\text { hypertension, or } \\
\text { benign breast or } \\
\text { gynaecological } \\
\text { tumours }\end{array}$ & $\begin{array}{l}11 \text { cases out of } \\
1809 \text { patients, } \\
\text { median follow-up } \\
\text { time } 29 \text { (range 0- } \\
\text { 47) months } \\
\text { TB incidence: } \\
307 / 100000 \\
\text { person-years }\end{array}$ & $\begin{array}{c}5 \text { cases out of } \\
1809 \text { patients } \\
\text { TB incidence: } \\
77 / 100000 \\
\text { person-years }\end{array}$ & $\begin{array}{c}\text { IRR } \\
4.69 \\
(1.52- \\
14.46)\end{array}$ & $\begin{array}{l}\text { controls matched } \\
\text { for age, sex and } \\
\text { comorbidities, } \\
\text { and IRR adjusted } \\
\text { for sex, } \\
\text { comorbidity and } \\
\text { presence of old } \\
\text { healed TB }\end{array}$ \\
\hline $\begin{array}{l}\text { Huang } 2011 \text { [29] } \\
2000-2006\end{array}$ & $\begin{array}{l}\mathrm{n}=2177 \text { cancer patients } \\
\text { at Cancer Center of } \\
\text { Taipei Veterans General } \\
\text { Hospital, Taiwan } \\
\text { median or mean age } \\
\text { and age range were not } \\
\text { specified; } \\
\text { excluded: prior } \\
\text { gastrectomy, prior TB } \\
\text { treatment, active TB } \\
\text { within } 6 \text { months before } \\
\text { the diagnosis of gastric } \\
\text { cancer and receiving } \\
\text { anti-TB treatment, } \\
\text { concurrent TB with } \\
\text { gastric cancer treatment } \\
\text { (+/-2 months) }\end{array}$ & $\begin{array}{c}\text { gastric cancer } \\
\text { (subtypes: } \\
\text { adenocarcinoma, } \\
\text { signet cell carcinoma, } \\
\text { mucinous } \\
\text { adenocarcinoma, } \\
\text { GIST, others) }\end{array}$ & not specified & $\begin{array}{c}\text { general } \\
\text { population in } \\
\text { Taiwan in } 2006\end{array}$ & $\begin{array}{l}44 \text { cases out of } \\
2177 \text { patients (5578 } \\
\text { person-years), } \\
\text { follow-up time } \\
\text { ranged from } 0 \text { to } \\
10 \text { years } \\
\text { Crude incidence: } \\
788 / 100000 \\
\text { person-years } \\
\text { Standardised } \\
\text { incidence: } \\
134.3(80.9-187.6) / \\
100000 \\
\text { person-years }\end{array}$ & $\begin{array}{c}\text { TB incidence: } \\
63.7 / 100000 \\
\text { population in } \\
2006\end{array}$ & $\begin{array}{c}\text { SIR } \\
2.11 \\
(1.57- \\
2.84)^{\pi}\end{array}$ & $\begin{array}{l}\text { TB incidence was } \\
\text { standardised for } \\
\text { age and sex }\end{array}$ \\
\hline $\begin{array}{c}\text { Kim 2014 [30] } \\
2003-2009\end{array}$ & $\begin{array}{l}\mathrm{n}=2684 \text { patients who } \\
\text { underwent gastrectomy } \\
\text { for gastric cancer, at a } \\
\text { tertiary referral hospital } \\
\text { in South Korea; } \\
\text { excluded: diagnosis of } \\
\text { concurrent pulmonary } \\
\text { TB, on TB treatment for } \\
3 \text { months before } \\
\text { gastrectomy }\end{array}$ & $\begin{array}{c}\text { gastric cancer: } \\
\text { adenocarcinoma } \\
\text { (81\%), signet-ring cell } \\
\text { carcinoma (17\%), } \\
\text { mucinous } \\
\text { adenocarcinoma }(2 \%), \\
\text { mixed carcinoma } \\
(0.4 \%), \text { GIST }(0.1 \%)\end{array}$ & $\begin{array}{c}\text { Information on } \\
\text { chemotherapy was } \\
\text { not available }\end{array}$ & $\begin{array}{c}\text { General } \\
\text { population of } \\
\text { South Korea }\end{array}$ & $\begin{array}{l}35 \text { cases out of } \\
2684 \text { patients, } \\
\text { median duration of } \\
\text { follow-up: } 4 \text { years } \\
\text { Crude incidence: } \\
327 / 100000 \\
\text { person-years } \\
\text { Age-standardised } \\
\text { incidence: } \\
404 / 100000 \\
\text { person-years }\end{array}$ & $\begin{array}{l}\text { TB incidence } \\
\text { in the general } \\
\text { population: } \\
78 / 100000 \text { in } \\
2010\end{array}$ & $\begin{array}{l}\text { SIR } 5.2 \\
(4.08- \\
6.62)\end{array}$ & $\begin{array}{c}\text { TB incidence was } \\
\text { standardised for } \\
\text { age }\end{array}$ \\
\hline
\end{tabular}




\begin{tabular}{|c|c|c|c|c|c|c|c|c|}
\hline $\begin{array}{l}\text { Study, year of } \\
\text { publication } \\
\text { [ref.] } \\
\text { Study period }\end{array}$ & Population and setting & Cancer type & Cancer treatment & $\begin{array}{l}\text { Control/general } \\
\text { population }\end{array}$ & $\begin{array}{l}\text { TB cases and } \\
\text { incidence in } \\
\text { cancer group }\end{array}$ & $\begin{array}{l}\text { TB cases and } \\
\text { incidence in } \\
\text { control group }\end{array}$ & $\begin{array}{l}\text { Rate } \\
\text { ratio } \\
{[95 \%} \\
\text { CI) }\end{array}$ & $\begin{array}{l}\text { Adjustment for } \\
\text { potential } \\
\text { confounders }\end{array}$ \\
\hline $\begin{array}{c}\text { Choi } 2015 \text { [31] } \\
\text { 2001-2008 }\end{array}$ & $\begin{array}{l}\mathrm{n}=1935 \text { patients with } \\
\text { early gastric cancer } \\
\text { stage IA, aged } 24 \text { to } \\
88 \text { years, identified from } \\
\text { the database of the } \\
\text { Center for Gastric } \\
\text { Cancer of the National } \\
\text { Cancer Center, Korea; } \\
\text { excluded: cases with TB } \\
\text { before or within } \\
3 \text { months after } \\
\text { gastrectomy }\end{array}$ & $\begin{array}{c}\text { gastric cancer, stage } \\
\text { IA }\end{array}$ & $\begin{array}{l}77.3 \% \text { had a } \\
\text { gastrectomy and } \\
22.7 \% \text { had an } \\
\text { endoscopic resection } \\
\text { of the gastric cancer }\end{array}$ & $\begin{array}{l}\text { General } \\
\text { population of } \\
\text { Korea in the } \\
\text { same age group } \\
\text { as the patients } \\
\text { with gastric } \\
\text { cancer }\end{array}$ & $\begin{array}{l}31 \text { cases out of } \\
1935 \text { patients ( } 9272 \\
\text { patient-years of } \\
\text { follow-up), median } \\
\text { follow-up time: } \\
4.9 \text { years } \\
\text { TB incidence: } \\
334 / 100000 \\
\text { person-years }\end{array}$ & $\begin{array}{c}\text { TB incidence: } \\
87 / 100000 \text { in } \\
2013\end{array}$ & $\begin{array}{l}\text { IRR } \\
\text { whole } \\
\text { cohort } \\
3.84^{\#} \\
(2.70- \\
5.46)^{7}\end{array}$ & $\begin{array}{c}\text { TB incidence was } \\
\text { standardised for } \\
\text { age }\end{array}$ \\
\hline $\begin{array}{l}\text { Fang 2015 [32] } \\
\text { 2000-2011 }\end{array}$ & $\begin{array}{l}\mathrm{n}=36972 \text { patients newly } \\
\text { diagnosed with gastric } \\
\text { cancer, aged } 20 \text { years } \\
\text { and older, identified from } \\
\text { the NHI database in } \\
\text { Taiwan; } \\
\text { excluded: patients } \\
\text { whose diagnosis of TB } \\
\text { occurred before } \\
\text { enrolment, patients who } \\
\text { were diagnosed with TB } \\
\text { within } 30 \text { days of the } \\
\text { diagnosis of gastric } \\
\text { cancer }\end{array}$ & gastric cancer & $\begin{array}{l}66.9 \% \text { of patients had } \\
\text { major surgery, } 52.2 \% \\
\text { received } \\
\text { chemotherapy, } 14.3 \% \\
\text { received radiotherapy }\end{array}$ & $\begin{array}{l}36972 \text { subjects } \\
\text { identified from } \\
\text { the NHI } \\
\text { database, } \\
\text { matched by age, } \\
\text { sex and } \\
\text { presence of } \\
\text { comorbidities }\end{array}$ & $\begin{array}{c}521 \text { cases out of } \\
36972 \text { patients; } \\
\text { no information on } \\
\text { median follow-up } \\
\text { time } \\
\text { TB incidence: } \\
523 / 100000 \\
\text { person-years }\end{array}$ & $\begin{array}{l}640 \text { cases out } \\
\text { of } 36972 \\
\text { matched } \\
\text { subjects in the } \\
\text { control cohort } \\
\text { TB incidence: } \\
344 / 100000 \\
\text { person-years }\end{array}$ & $\begin{array}{c}\text { Crude } \\
\text { HR } 1.50 \\
(1.33- \\
1.68) \\
\text { aHR } \\
1.59 \\
(1.41- \\
1.79)\end{array}$ & $\begin{array}{c}\text { controls matched } \\
\text { by age, sex and } \\
\text { comorbidities, } \\
\text { and HR adjusted } \\
\text { for age, sex and } \\
\text { comorbidities in } \\
\text { multivariate } \\
\text { analysis }\end{array}$ \\
\hline $\begin{array}{l}\text { Jung } 2016 \text { [33] } \\
\text { 2007-2009 }\end{array}$ & $\begin{array}{l}\mathrm{n}=1776 \text { patients who } \\
\text { underwent gastrectomy } \\
\text { for gastric cancer, aged } \\
20 \text { to } 89 \text { years, at a } \\
\text { tertiary referral hospital } \\
\text { in Seoul, South Korea; } \\
\text { excluded: patients who } \\
\text { received chemotherapy } \\
\text { after gastrectomy, } \\
\text { patients who had a } \\
\text { history of previous } \\
\text { gastrectomy, patients } \\
\text { who were treated for TB } \\
\text { within } 6 \text { months before } \\
\text { surgery or developed TB } \\
\text { within } 2 \text { months after } \\
\text { surgery, patients who } \\
\text { visited the hospital only } \\
\text { once after surgery }\end{array}$ & gastric cancer & $\begin{array}{l}\text { Patients who } \\
\text { received } \\
\text { chemotherapy after } \\
\text { gastrectomy were } \\
\text { excluded }\end{array}$ & $\begin{array}{c}\text { General } \\
\text { population of } \\
\text { South Korea }\end{array}$ & $\begin{array}{c}16 \text { cases out of } \\
1776 \text { patients } \\
\text { (7152.5 } \\
\text { patient-years), } \\
\text { mean duration of } \\
\text { follow-up: } 4 \text { years } \\
\text { TB incidence: } \\
423.7 / 100000 \\
\text { person-years }\end{array}$ & $\begin{array}{l}\text { TB incidence: } \\
89.4 / 100000 \\
\text { person-years } \\
\text { in } 2008\end{array}$ & $\begin{array}{c}\text { SIR } \\
2.22 \\
(1.27- \\
3.60)\end{array}$ & $\begin{array}{l}\text { TB incidence was } \\
\text { standardised for } \\
\text { age and sex }\end{array}$ \\
\hline
\end{tabular}


TABLE 1 Continued

\begin{tabular}{|c|c|c|c|c|c|c|c|c|}
\hline $\begin{array}{l}\text { Study, year of } \\
\text { publication } \\
\text { [ref.] } \\
\text { Study period }\end{array}$ & Population and setting & Cancer type & Cancer treatment & $\begin{array}{c}\text { Control/general } \\
\text { population }\end{array}$ & $\begin{array}{l}\text { TB cases and } \\
\text { incidence in } \\
\text { cancer group }\end{array}$ & $\begin{array}{l}\text { TB cases and } \\
\text { incidence in } \\
\text { control group }\end{array}$ & $\begin{array}{l}\text { Rate } \\
\text { ratio } \\
(95 \% \\
\text { CI) }\end{array}$ & $\begin{array}{l}\text { Adjustment for } \\
\text { potential } \\
\text { confounders }\end{array}$ \\
\hline $\begin{array}{l}\text { Alhashimi } 1988 \\
\text { [34] } \\
1975-82\end{array}$ & $\begin{array}{c}\mathrm{n}=257 \text { cancer patients } \\
\text { (89 }(35 \%) \text { with latent TB } \\
\text { infection based on } \\
\mathrm{TST} \geqslant 10 \mathrm{~mm} \text { ) without } \\
\text { isoniazid prophylaxis, } \\
\text { mean age } 58.5 \text { years } \\
\text { (range } 40-69 \text { years) } \\
\text { VA Medical Center, } \\
\text { Washington DC, USA }\end{array}$ & lung cancer & $\begin{array}{l}\text { all patients received } \\
\text { chemotherapy with } \\
\text { or without } \\
\text { radiotherapy, } \\
\text { corticosteroid } \\
\text { therapy or both }\end{array}$ & $\begin{array}{c}\text { residents of } \\
\text { Washington, DC, } \\
\text { USA, } \geqslant 45 \text { years }\end{array}$ & $\begin{array}{c}3 \text { cases out of } 257 \\
\text { patients; no } \\
\text { information on } \\
\text { median follow-up } \\
\text { time } \\
\text { TB incidence: } \\
1100 / 100000 / \text { year }\end{array}$ & $\begin{array}{c}\text { number of TB } \\
\text { cases and size } \\
\text { of control } \\
\text { population not } \\
\text { given } \\
\text { TB incidence: } \\
73 / 100000 / \\
\text { year }\end{array}$ & $\begin{array}{l}\text { IRR } \\
15.00^{\#} \\
(4.84- \\
46.51)^{\pi}\end{array}$ & $\begin{array}{l}\text { used the same or } \\
\text { a similar age } \\
\text { group in the } \\
\text { general } \\
\text { population for } \\
\text { comparison }\end{array}$ \\
\hline $\begin{array}{l}\text { Wessels } 1992 \\
\text { [35] } \\
1983-1990\end{array}$ & $\begin{array}{c}\mathrm{n}=278 \text { patients registered } \\
\text { in the Tygerberg } \\
\text { Hospital's children's } \\
\text { cancer registry } \\
\text { (below the age of } \\
15 \text { years); } \\
\text { excluded: children with } \\
\text { brain tumours and those } \\
\text { who died within } 6 \text { weeks } \\
\text { of cancer diagnosis }\end{array}$ & $\begin{array}{l}\text { haematological and } \\
\text { solid (excluding brain } \\
\text { tumours) }\end{array}$ & not specified & $\begin{array}{c}\text { general } \\
\text { population of } \\
\text { South Africa and } \\
\text { the Western } \\
\text { Cape in } 1986\end{array}$ & $\begin{array}{l}13 \text { cases out of } 278 \\
\text { patients (277.5 } \\
\text { patient-years of } \\
\text { follow-up); no } \\
\text { information on } \\
\text { median follow-up } \\
\text { time } \\
\text { TB incidence: } \\
4150 / 100000 \\
\text { person-years of } \\
\text { follow-up }\end{array}$ & $\begin{array}{l}\text { TB incidence: } \\
360 / 100000 \\
\text { among } \\
\text { children aged } \\
\text { below } 15 \text { years }\end{array}$ & $\begin{array}{c}\text { SIR } \\
11.53 \\
(6.69- \\
19.85)\end{array}$ & $\begin{array}{l}\text { used the same or } \\
\text { a similar age } \\
\text { group in the } \\
\text { general } \\
\text { population for } \\
\text { comparison }\end{array}$ \\
\hline $\begin{array}{l}\text { Stefan 2008 [36] } \\
1991-2005\end{array}$ & $\begin{array}{c}\mathrm{n}=625 \text { children (0-15 } \\
\text { years old) undergoing } \\
\text { cancer therapy at } \\
\text { Tygerberg Hospital, } \\
\text { located in a Cape Town } \\
\text { area with high TB } \\
\text { prevalence; } \\
\text { mean age at cancer } \\
\text { diagnosis was } 5.1 \text { years } \\
\text { (median 3.8, range } \\
0-15 \text { ); } \\
\text { excluded: children who } \\
\text { completed TB treatment } \\
\text { before the cancer } \\
\text { diagnosis }\end{array}$ & $\begin{array}{l}\text { haematological and } \\
\text { solid }\end{array}$ & not specified & $\begin{array}{l}\text { general } \\
\text { population of } \\
\text { South Africa } \\
\text { aged } 0-15 \text { years }\end{array}$ & $\begin{array}{c}57 \text { cases out of } 625 \\
\text { patients; no } \\
\text { information on } \\
\text { median follow-up } \\
\text { time } \\
\text { Crude incidence: } \\
9135 / 100000 / \text { year }\end{array}$ & $\begin{array}{l}\text { TB incidence: } \\
407 / 100000 / \\
\text { year among } \\
\text { children aged } \\
0-15 \text { years }\end{array}$ & $\begin{array}{c}\text { IRR } \\
22.44 \\
(17.31- \\
29.10)^{9}\end{array}$ & $\begin{array}{l}\text { used the same or } \\
\text { a similar age } \\
\text { group in the } \\
\text { general } \\
\text { population for } \\
\text { comparison }\end{array}$ \\
\hline
\end{tabular}

TB: tuberculosis; NHI: National Health Insurance; IRR: incidence rate ratio; alRR: adjusted incidence rate ratio; SIR: standardised incidence ratio; CML: chronic myeloid leukaemia; CLL: chronic lymphocytic leukaemia; SAA: severe aplastic anaemia; MDS: myelodysplastic syndrome; HR: hazard ratio; aHR: adjusted hazard ratio; GIST: gastrointestinal stromal tumour; TST:

tuberculin skin test." : IRR was not specified in the original study, but was calculated by dividing the incidence of TB in cancer patients by the incidence of TB in the reference group.

ף: Confidence interval was estimated using the Poisson assumption. 


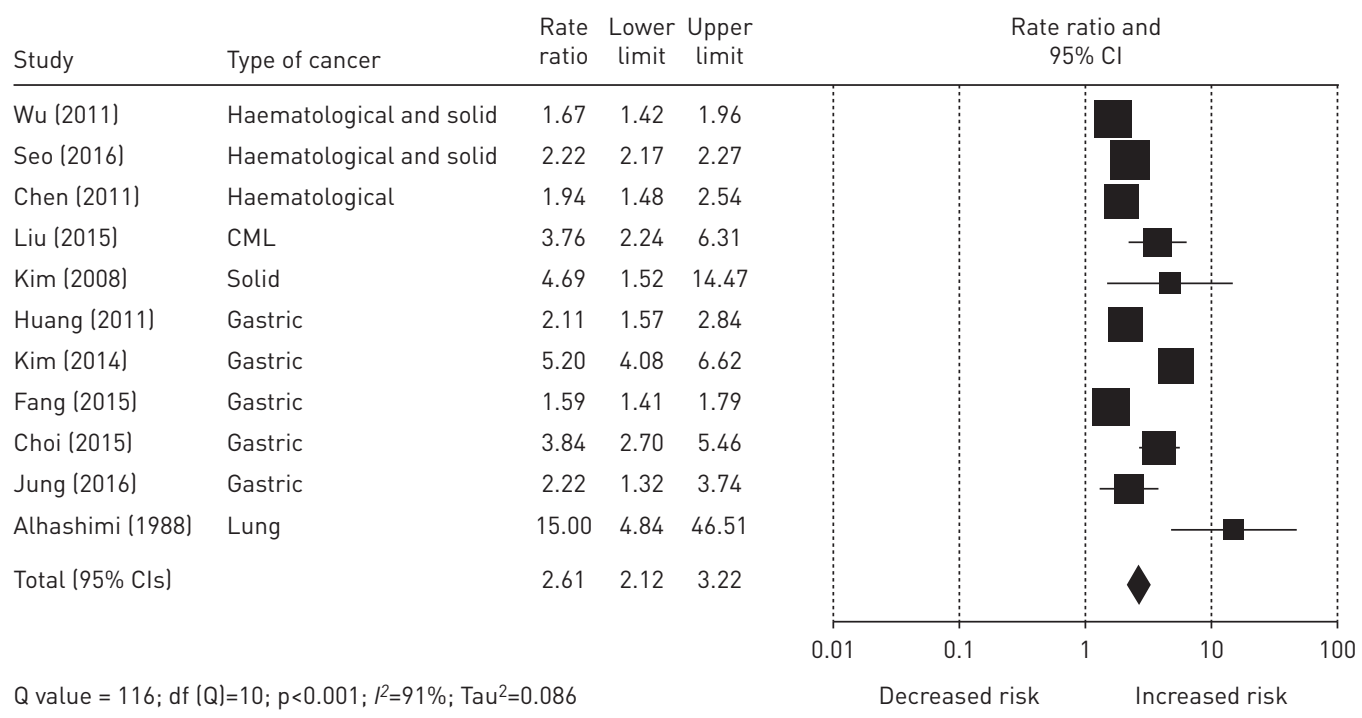

FIGURE 2 Forest plot of relative risk of tuberculosis in all adult cancer studies. CML: chronic myeloid leukaemia.

studies performed in children with haematological and solid malignancies was 16.82 (95\% CI 8.81-32.12; $Q=5, d f=1, \mathrm{p}=0.030 ; I^{2}=79 \%$ ) (figure 3).

\section{Subgroup analysis in adult cancer patients}

Separate analysis of haematological malignancies and solid tumours (excluding studies that did not present measures of effect size for these two groups separately) yielded an IRR of 3.53 (95\% CI 1.63-7.64; $Q=82$, $d f=3, \mathrm{p}<0.001 ; I^{2}=96 \%$ ) (figure 4) for haematological malignancies, and an IRR of 2.25 (95\% CI 1.96-2.58; $Q=341, d f=29, p<0.001 ; I^{2}=91 \%$ ) (figure 5) for solid cancers.

Of the solid tumours for which data was available from at least three studies, lung cancer had an IRR of 6.14 (95\% CI 1.97-19.20; $I^{2}=76 \%$ ) (figure 6a), gastric cancer had an IRR of 2.63 (95\% CI 1.96-3.52; $I^{2}=93 \%$ ) (figure $6 \mathrm{~b}$ ), breast cancer had an IRR of 2.17 (95\% CI 1.98-2.38; $I^{2}=0 \%$ ) (figure 6c), liver cancer had an IRR of 2.02 (95\% CI $0.83-4.91 ; I^{2}=83 \%$ ) (figure 6d) and colon cancer had an IRR of 2.00 (95\% CI $1.16-3.43 ; I^{2}=75 \%$ ) (figure $6 \mathrm{e}$ ).

\section{Effect of surgery, chemotherapy and/or radiotherapy on TB risk}

The majority of reviewed studies provided no detailed information on the number of patients with cancer who had undergone surgery, chemotherapy and/or radiotherapy, and the temporal relationship between treatment and a diagnosis of TB.

Treatment details were available for a number of studies in gastric cancer. A nationwide study from Taiwan did not establish any treatment modality (gastrectomy, chemotherapy or radiotherapy) as an independent risk factor for TB in patients with gastric cancer [32]. Another study from Taiwan found that chemotherapy was associated with a higher risk for $\mathrm{TB}$, compared to gastrectomy and palliative therapy in patients with gastric cancer (SIR 2.91, 2.50 and 1.19, respectively) [29]. A nationwide Korean study in patients with early gastric cancer showed that patients who underwent endoscopic resection showed no increase in the relative risk of TB, compared to the general population; whereas patients who underwent gastrectomy had a hazard ratio of 7.92 (95\% CI 1.08-58.16) for the risk of developing TB [31]. Another Korean study found that patients undergoing total gastrectomy had an OR of 3.48 (95\% CI 1.25-9.66) for the risk of developing TB, compared to patients who underwent subtotal gastrectomy [33].

\section{Assessment of risk of bias of included studies}

The risk of bias assessment, using the Newcastle-Ottawa Quality Assessment Scale for Cohort Studies [20], showed that this risk was moderate to low (supplementary Table S1). Risk of bias was mainly related to selection of the reference group (control cohort, not drawn from the same community, during the same period as the cancer cohort), as well as the comparability of the reference group (lack of adjustment/ stratification for the most important confounders) and uncertainties regarding the reporting of follow-up. Heterogeneity between studies for all outcomes under evaluation was high, although it was reduced during sub-group analysis for different solid organ tumours (but remained high for most subgroups). 


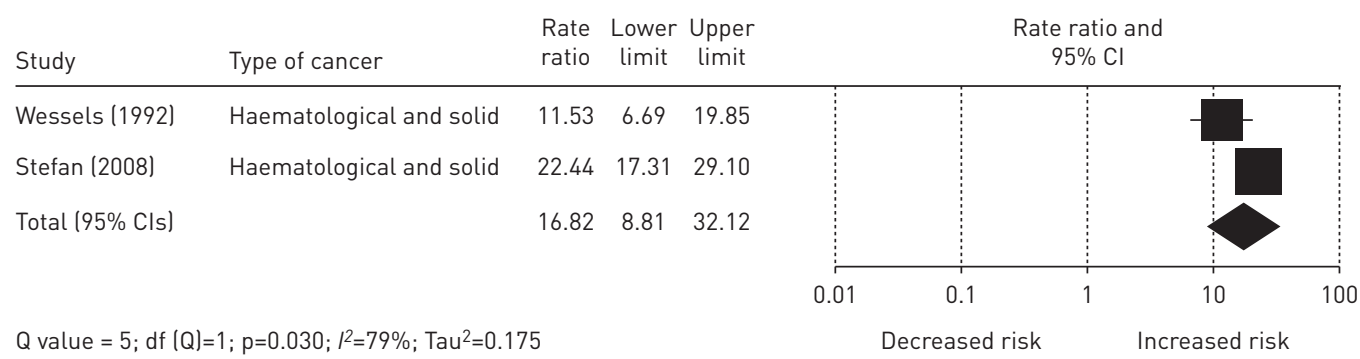

FIGURE 3 Forest plot of relative risk of tuberculosis in children with cancer.

Only one study provided a prevalence estimate of LTBI, based on the TST/IGRA in the study cohort (89/ 257, 35\%), and stated that five patients received LTBI treatment [34]. These patients were, however, excluded from the analysis. No other study indicated whether participants received treatment for LTBI. Six studies, four of which evaluated TB exclusively in gastric cancer, included information on the history of TB treatment and/or radiological evidence of previously healed TB [28-31,33,34], with a prevalence of previous $\mathrm{TB}$ on chest radiographs ranging from 7\% [34] to $17 \%$ [28] in the study population. There was a paucity of available information on HIV status. One paediatric study from South Africa reported that six out of 57 children with cancer and TB (11\%) were infected with HIV [36]. Two studies excluded HIV-positive individuals $[26,28]$. The remainder of the studies disclosed no information on HIV status.

\section{Discussion}

The meta-analysis showed a statistically significantly increased risk of TB in cancer patients, compared to the general population. Most solid cancers, for which data from three or more studies were available, were associated with an approximately two-fold increase in the risk of developing TB, compared to the general population. This increase was significant for gastric, breast and colon cancer, but not for liver cancer (IRR 2.02; 95\% CI 0.83-4.91). Lung cancer was associated with a six-fold increase in TB. The relative risk of TB in gastric cancer (IRR 2.63, 95\% CI 1.96-3.52), which is often treated with gastrectomy (associated with malnutrition [44], a risk factor for TB [45, 46]), was not markedly different from the relative risk in other solid cancers. The relative risk of TB in haematological cancers in adults (IRR 3.53; 95\% CI 1.63-7.64) was only moderately higher than that in adults with solid cancers (IRR 2.25; 95\% CI 1.96-2.58). The highest relative risk of TB was found in children with haematological malignancies or solid cancers (IRR 16.82, 95\% CI 8.81-32.12).

To our knowledge, this is the first meta-analysis to assess the relative risk of TB in haematological malignancies and solid cancers, among studies that include information on a comparator group (controls or general population of the study setting and period). Although significant heterogeneity was noted between included studies, the random effects model that was applied yields estimates that appropriately reflect this variation (i.e. via the 95\% CIs). Thus, the pooled estimate is not as important as the upper and lower $95 \% \mathrm{CI}$ of the estimates, in consideration of whether the relative risk is too low (based on the upper 95\% CI) to warrant LTBI screening, or sufficiently high (based on the lower 95\% CI) to consider LTBI screening.

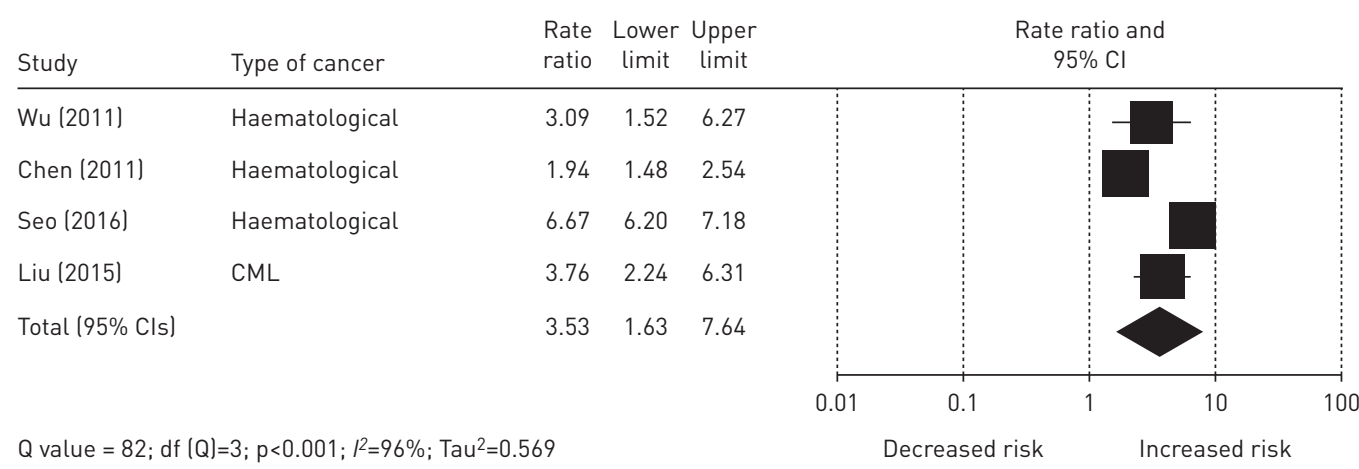

FIGURE 4 Forest plot of relative risk of tuberculosis in adults with haematological malignancies. CML: chronic myeloid leukaemia. 


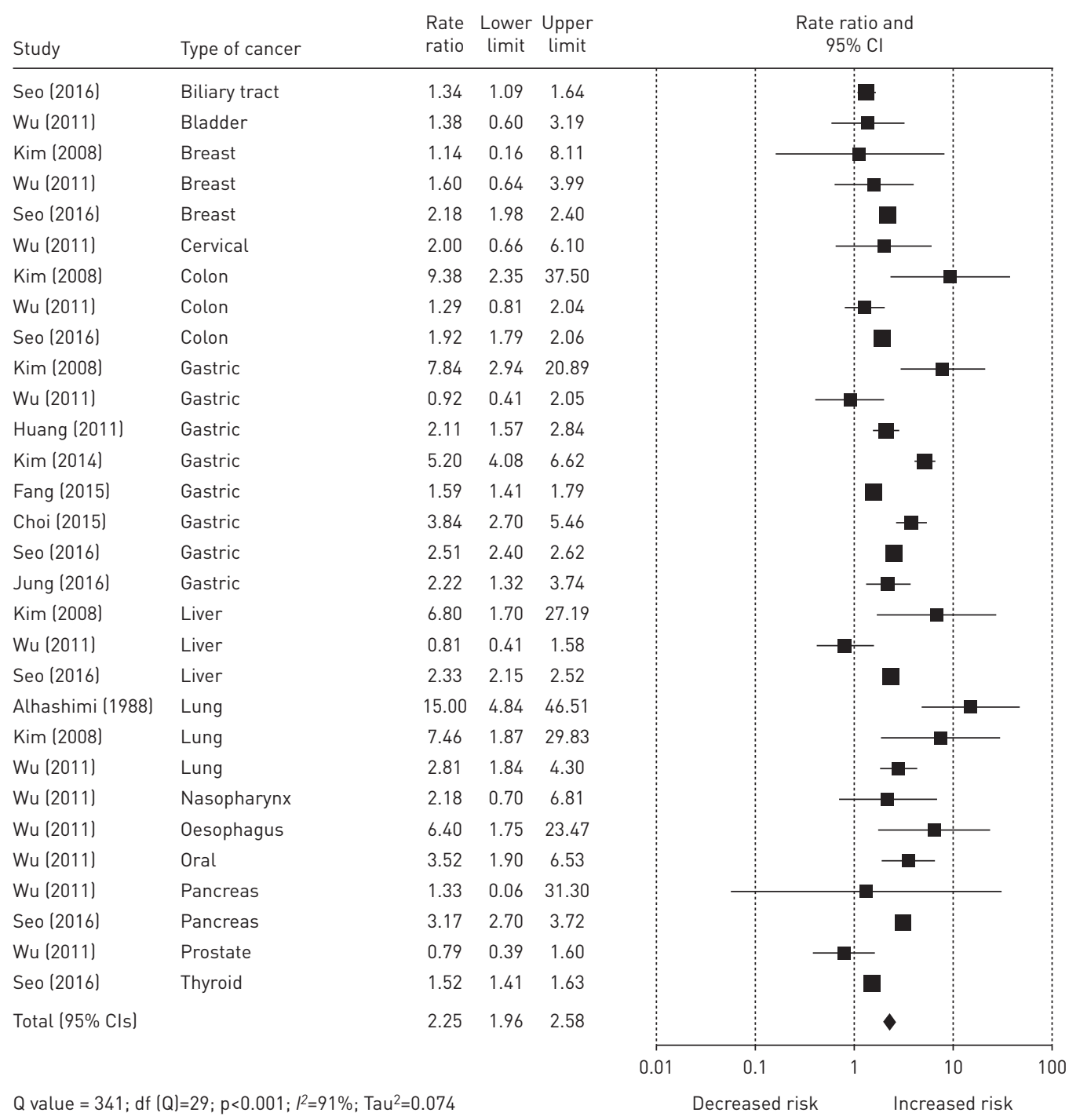

FIGURE 5 Forest plot of relative risk of tuberculosis in adults with different solid tumours.

Children with solid cancers and haematological malignancies have a high relative risk of developing TB, and should be considered for systematic screening and treatment of LTBI. Although both of the included paediatric studies were conducted in South Africa, a setting with a high risk for reinfection with TB, this should not have affected the estimated relative risk of $\mathrm{TB}$, as the control population shared the same risk of exposure. The implementation of LTBI screening and treatment, however, depending on the setting and our recommendations, only applies to high or upper middle income countries with a TB incidence of $<100$ per 100000 per year [9].

The findings of this meta-analysis facilitate comparison with the relative risk of TB among other groups with an increased risk of developing TB, and inform screening recommendations for latent TB infection and TB chemoprophylaxis in patients with cancer. The upper $95 \%$ CI of the relative risk (RR) of TB in cancer (3.22) is lower than the relative risk in groups currently targeted for LTBI screening and treatment, such as patients with HIV (RR of 50-110 [47, 48]), contacts of patients with active TB (RR of 10.4 during follow-up of 90 days to 2 years after initial healthcare contact, with $1.5 \%$ of all TB contacts already diagnosed with TB at initial screening [49]), patients with chronic renal failure (RR of 7.8[5]) and patients being treated with TNF- $\alpha$ inhibitors (RR 1.8-29.3 [50]). The reactivation of TB can occur at any time after initial infection [3], and the estimated cumulative lifetime risk to develop TB is therefore important to inform decisions about LTBI screening and treatment. In chronic conditions, such as diabetes and chronic renal failure, the increased risk associated with the disease can be expected to last a lifetime, without any considerable reduction in life expectancy due to the underlying disease. In cancer, however, immunosuppressive states are more likely to be temporary (e.g. during chemotherapy). In addition, life 


\begin{tabular}{|c|c|c|c|c|}
\hline $\begin{array}{l}\text { a) } \\
\text { Study }\end{array}$ & Type of cancer & $\begin{array}{l}\text { Rate } \\
\text { ratio }\end{array}$ & $\begin{array}{l}\text { Lower } \\
\text { limit }\end{array}$ & $\begin{array}{c}\text { Upper } \\
\text { limit }\end{array}$ \\
\hline Alhashimi (1988) & Lung & 15.00 & 4.84 & 46.51 \\
\hline Kim (2008) & Lung & 7.46 & 1.87 & 29.83 \\
\hline Wu (2011) & Lung & 2.81 & 1.84 & 4.30 \\
\hline Total (95\% Cls) & & 6.14 & 1.97 & 19.20 \\
\hline
\end{tabular}

$Q$ value $=8 ; \mathrm{df}(\mathrm{Q})=2 ; \mathrm{p}=0.014 ; l^{2}=76 \% ; \mathrm{Tau}^{2}=0.756$

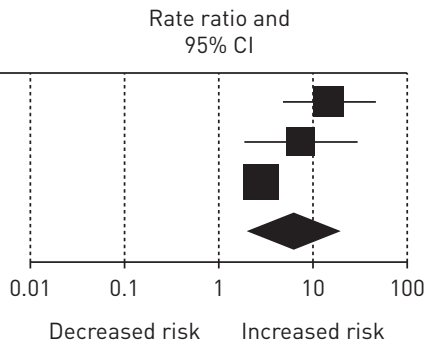

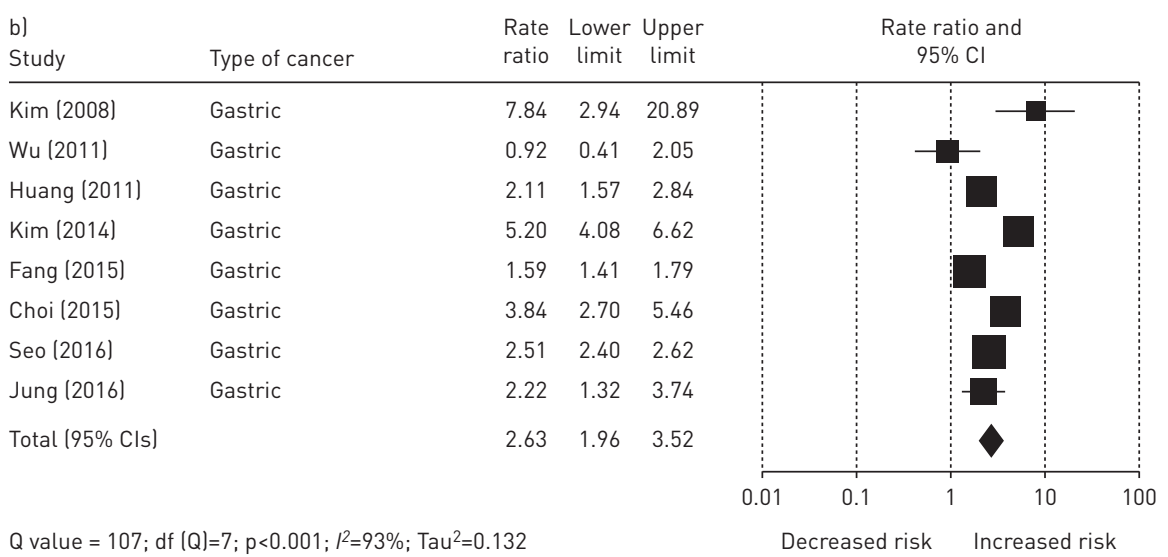

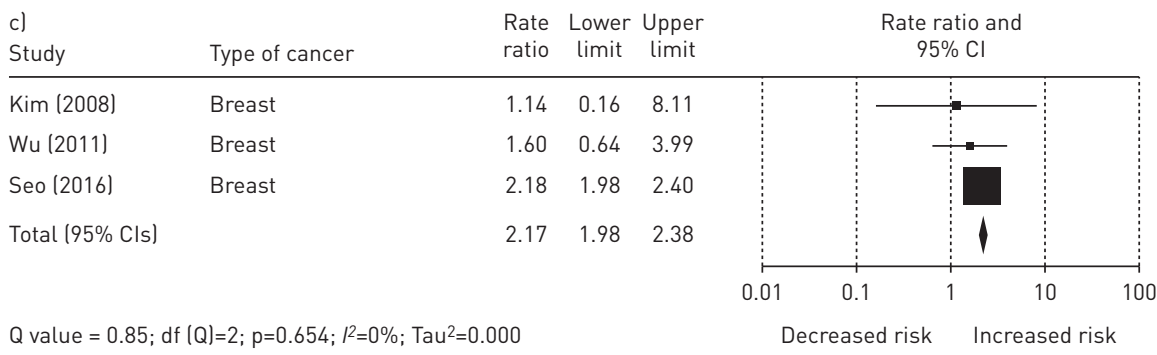

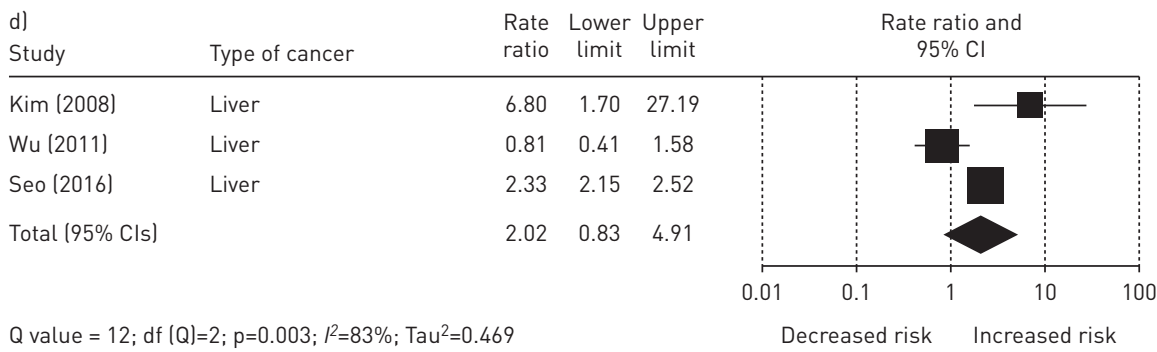

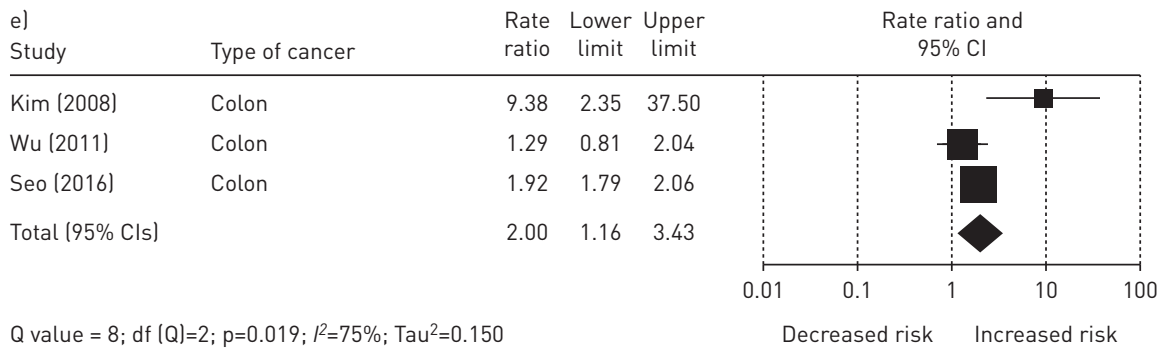

FIGURE 6 a) Forest plot of relative risk of tuberculosis in persons with lung cancer. b) Forest plot of relative risk of tuberculosis in persons with gastric cancer. c) Forest plot of relative risk of tuberculosis in persons with breast cancer. d) Forest plot of relative risk of tuberculosis in persons with liver cancer. e) Forest plot of relative risk of tuberculosis in persons with colon cancer. 
expectancy is markedly reduced in many cancers, which reduces the cumulative lifetime risk of developing TB. This has to be considered when comparing the relative risk of TB in cancer patients with that in other immunocompromised groups. Based on these considerations, we suggest that in adult patients with cancer, there is not a sufficiently increased risk of TB to recommend systematic screening and treatment for LTBI, independent of other risk factors. As outlined in the WHO guidelines, the potential harms and benefits of LTBI treatment will need to be weighed on an individual patient basis [9]. The introduction of systematic screening and treatment of LTBI in any group needs to take into account the local context, accessibility, equity, cost and implementation aspects [9]; however, discussion of these aspects is beyond the scope of the present research.

The overall incidence rate (per year) of TB decreases with the time that has elapsed since the diagnosis of cancer (associated with early treatment). This was demonstrated in the largest study that was included in the present meta-analysis (including 855382 patients with cancer), which showed that the relative risk of TB was highest, immediately after the diagnosis of cancer, and gradually declined over time with a SIR of 3.70 (95\% CI 3.57-3.83) for the first 6 months; SIR of 2.19 (95\% CI 2.08-2.30) for months 6 to 11; SIR of 1.75 (95\% CI 1.67-1.84) for months 12 to 23; and SIR of 1.43 (95\% CI 1.36-1.51) for greater than 24 months after diagnosis [25]. In addition to the impaired immunity due to cancer and cancer treatment, more intense medical follow-up and examinations early in the course of cancer disease might result in an increase in the number of diagnosed TB cases. This might result in overestimation of the risk of $\mathrm{TB}$ in cancer patients.

Considerations regarding the relative risk of TB during different periods following the diagnosis of cancer are also relevant for interpretation of the present results. Studies with a prolonged follow-up of cancer patients will yield a lower incidence estimate per person-years of follow-up, as the risk of TB would decline with time elapsed since diagnosis. This risk reduction over time was especially prominent in haematological malignancies in the aforementioned large cohort study, in which the SIR was 12.01 (95\% CI 10.81-13.30) for the first 6 months following diagnosis, and decreased to a SIR of $2.70(2.12-3.39)$ for greater than 24 months after diagnosis [25]. In the present meta-analysis, the pooled IRR in haematological malignancies (in patients aged 15 years or above) of 3.53 (95\% CI 1.63-7.64) might have been lower than expected, because of a prolonged follow-up. The TB incidence rates showed a decline with increased follow-up duration, reflecting the reduction in TB risk after initial diagnosis and completion of chemotherapy [51].

One strength of our systematic review was its rigorous methodology, in terms of the inclusion only of studies that provided an incidence rate of TB (rather than a cumulative incidence or prevalence) in patients with cancer, as well as a TB incidence rate in a control/general population. One limitation of the present systematic review was its inability to differentiate between risk arising from cancer per se and that from cancer treatment. The available data also did not allow us to consider the impact of cancer stage. As treatment modalities are chosen based on the cancer stage and expected prognosis, and the follow-up period is shorter in patients with advanced cancer, it is very difficult, if not impossible, to establish the independent effect of treatment modalities and cancer stage on the risk of TB reactivation from cohort studies. As outlined above, variable follow-up times in different studies are likely to have contributed to differences in estimates of TB risk. The included studies did not distinguish between TB caused by recent transmission and TB secondary to reactivation, with only the latter being an indication for LTBI screening.

The use of different reference groups (ranging from matched controls to the unadjusted general population of the study setting) could have added to the heterogeneity identified in the meta-analysis. The lowest quality reference group was the general population of the study setting, without adjustment for potential confounders, particularly age. This could have led to overestimation of the relative risk of TB in populations with a higher incidence of $\mathrm{TB}$ among older people (the more likely scenario), or underestimation of the relative risk of TB in populations with a higher incidence of TB among younger people. However, only one study did not adjust for age as a potential confounder [26].

In summary, this meta-analysis showed that patients with cancer have an increased risk of developing TB, compared to the general population. Children with solid cancers or haematological malignancies have a high relative risk of developing $\mathrm{TB}$, and should be considered for systematic screening and treatment of LTBI, especially when they originate from settings with a high TB incidence. In adults with cancer, the relative risk of TB was only modestly increased. The slightly increased risk, paired with the fact that, in many instances, the risk decreases over time since the initial cancer diagnosis, and/or the cumulative lifetime risk is reduced because of reduced life expectancy, suggests that adult patients with cancer do not require systematic screening and treatment for LTBI, independent of other risk factors. Adults with cancer, especially those with haematological malignancies, who have additional risk factors (such as being a migrant from a country with a high TB burden), should be considered for LTBI screening and treatment 
on a case-by-case basis. Among the solid cancers, lung cancer has one of the highest risks of TB, but prognosis is often poor [52], and a short mean expected survival might suggest that the benefits of LTBI treatment do not outweigh the associated inconvenience and risks in many patients. While current guidelines describe patients with gastrectomy as a high-risk group for TB, our analysis (which included a number of recent studies on gastric cancer and gastrectomy) showed no marked difference in the risk of TB in patients with gastric cancer, with or without gastrectomy, compared to patients with other solid cancers.

\section{References}

1 World Health Organization. Global Tuberculosis Report 2016. www.who.int/tb/publications/global_report/en/ Date last accessed: January 3, 2017.

2 Lonnroth K, Migliori GB, Abubakar I, et al. Towards tuberculosis elimination: an action framework for low-incidence countries. Eur Respir J 2015; 45: 928-952.

3 American Thoracic Society. Diagnostic standards and classification of tuberculosis. Am Rev Respir Dis 1990; 142: $725-735$.

4 Getahun H, Gunneberg C, Granich R, et al. HIV infection-associated tuberculosis: the epidemiology and the response. Clin Infect Dis 2010; 50: Suppl. 3, S201-S207.

5 Dobler CC, McDonald SP, Marks GB. Risk of tuberculosis in dialysis patients: a nationwide cohort study. PLoS One 2011; 6: e29563.

6 Dobler CC, Flack JR, Marks GB. Risk of tuberculosis among people with diabetes mellitus: an Australian nationwide cohort study. BMJ Open 2012; 2: e000666.

7 Dobler CC. Biologic agents and tuberculosis. Microbiol Spectr 2016; 4. doi: 10.1128/microbiolspec.TNMI7-00262016

8 Dobler CC. Biological agents and tuberculosis: risk estimates and screening strategies. Int J Rheum Dis 2015; 18: 264-267.

9 Getahun H, Matteelli A, Abubakar I, et al. Management of latent Mycobacterium tuberculosis infection: WHO guidelines for low tuberculosis burden countries. Eur Respir J 2015; 46: 1563-1576.

10 Smieja MJ, Marchetti CA, Cook DJ, et al. Isoniazid for preventing tuberculosis in non-HIV infected persons. Cochrane Database Syst Rev 2000; (2): CD001363.

11 National Institute for Health and Care Excellence (NICE). Tuberculosis [NG33], section 1.2.4 Managing latent TB in all age groups. January 2016. www.nice.org.uk/guidance/ng33/chapter/Recommendations\#latent-tb Date last accessed: January 3, 2017. Date last updated: May, 2016.

12 Targeted tuberculin testing and treatment of latent tuberculosis infection. Am J Respir Crit Care Med 2000; 161: S221-S247.

13 Feld R, Bodey GP, Groschel D. Mycobacteriosis in patients with malignant disease. Arch Intern Med 1976; 136: 67-70.

14 Kaplan MH, Armstrong D, Rosen P. Tuberculosis complicating neoplastic disease. A review of 201 cases. Cancer 1974; 33: 850-858.

15 Cheng MP, Abou Chakra CN, Yansouni CP, et al. Risk of active tuberculosis in patients with cancer: a systematic review and meta-analysis. Clin Infect Dis 2017; 64: 635-644.

16 Kamboj M, Sepkowitz KA. The risk of tuberculosis in patients with cancer. Clin Infect Dis 2006; 42: 1592-1595.

17 Christopoulos A, Saif MW, Sarris EG, et al. Epidemiology of active tuberculosis in lung cancer patients: a systematic review. Clin Respir J 2014; 8: 375-381.

18 Liang HY, Li XL, Yu XS, et al. Facts and fiction of the relationship between preexisting tuberculosis and lung cancer risk: a systematic review. Int J Cancer 2009; 125: 2936-2944.

19 Liberati A, Altman DG, Tetzlaff J, et al. The PRISMA statement for reporting systematic reviews and meta-analyses of studies that evaluate health care interventions: explanation and elaboration. PLoS Med 2009; 6: e1000100.

20 Wells G, Shea B, O'Connell D, et al. The Newcastle-Ottawa Scale (NOS) for assessing the quality of nonrandomised studies in meta-analyses. http://www.ohri.ca/programs/clinical_epidemiology/oxford.asp

21 Rothman, KJ., Greenland, S. Modern Epidemiology. 2nd Edn. Philadelphia, Lippincott, 1998.

22 Hernan MA. The hazards of hazard ratios. Epidemiology 2010; 21: 13-15.

23 DerSimonian R, Laird N. Meta-analysis in clinical trials. Control Clin Trials 1986; 7: 177-188.

$24 \mathrm{Wu} \mathrm{CY}, \mathrm{Hu} \mathrm{HY}, \mathrm{Pu} \mathrm{CY}$, et al. Aerodigestive tract, lung and haematological cancers are risk factors for tuberculosis: an 8-year population-based study. Int J Tuberc Lung Dis 2011; 15: 125-130.

25 Seo GH, Kim MJ, Seo S, et al. Cancer-specific incidence rates of tuberculosis: A 5-year nationwide population-based study in a country with an intermediate tuberculosis burden. Medicine (Baltim) 2016; 95 : e4919.

26 Chen CY, Sheng WH, Cheng A, et al. Clinical characteristics and outcomes of Mycobacterium tuberculosis disease in adult patients with hematological malignancies. BMC Infect Dis 2011; 11: 324

27 Liu CJ, Hong YC, Teng CJ, et al. Risk and impact of tuberculosis in patients with chronic myeloid leukemia: a nationwide population-based study in Taiwan. Int J Cancer 2015; 136: 1881-1887.

28 Kim HR, Hwang SS, Ro YK, et al. Solid-organ malignancy as a risk factor for tuberculosis. Respirology 2008; 13: 413-419.

29 Huang SF, Li CP, Feng JY, et al. Increased risk of tuberculosis after gastrectomy and chemotherapy in gastric cancer: a 7-year cohort study. Gastric Cancer 2011; 14: 257-265.

$30 \mathrm{Kim} \mathrm{CH}$, Im KH, Yoo SS, et al. Comparison of the incidence between tuberculosis and nontuberculous mycobacterial disease after gastrectomy. Infection 2014; 42: 697-704.

31 Choi IJ, Kim YW, Lee HS, et al. Risk factors for TB in patients with early gastric cancer: Is gastrectomy a significant risk factor for TB? Chest 2015; 148: 774-783.

32 Fang WL, Hung YP, Liu CJ, et al. Incidence of and risk factors for tuberculosis (TB) in gastric cancer patients in an area endemic for TB: A nationwide population-based matched cohort study. Medicine (Baltim) 2015; 94 : e2163. 
33 Jung WJ, Park YM, Song JH, et al. Risk factors for tuberculosis after gastrectomy in gastric cancer. World $J$ Gastroenterol 2016; 22: 2585-2591.

34 Alhashimi MM, Citron ML, Fossieck BE, Jr, et al. Lung cancer, tuberculin reactivity, and isoniazid. South Med J 1988; 81: 337-340.

35 Wessels G, Hesseling PB, Gie RP, et al. The increased risk of developing tuberculosis in children with malignancy. Ann Trop Paediatr 1992; 12: 277-281.

36 Stefan DC, Kruis AL, Schaaf HS, et al. Tuberculosis in oncology patients. Ann Trop Paediatr 2008; 28: 111-116.

37 Libshitz HI, Pannu HK, Elting LS, et al. Tuberculosis in cancer patients: an update. J Thorac Imaging 1997; 12: 41-46.

38 Ibrahim EM, Uwaydah A, al-Mulhim FA, et al. Tuberculosis in patients with malignant disease. Indian J Cancer 1989; 26: 53-57.

39 El-Sharif A, Afifi S, El-Dahshan R, et al. Characterization of Mycobacterium tuberculosis isolated from cancer patients with suspected tuberculosis infection in Egypt: identification, prevalence, risk factors and resistance pattern. Clin Microbiol Infect 2012; 18: E438-E445.

40 Karnak D, Kayacan O, Beder S. Reactivation of pulmonary tuberculosis in malignancy. Tumori 2002; 88: 251-254.

41 Suzuki Y, Imokawa S, Sato J, et al. Cumulative incidence of tuberculosis in lung cancer patients in Japan: A 6-year observational study. Respir Investig 2016; 54: 179-183.

42 Ortbals DW, Marr JJ. A comparative study of tuberculous and other mycobacterial infections and their associations with malignancy. Am Rev Respir Dis 1978; 117: 39-45.

43 Simonsen DF, Farkas DK, Sogaard M, et al. Tuberculosis and risk of cancer: a Danish nationwide cohort study. Int J Tuberc Lung Dis 2014; 18: 1211-1219.

44 Scholmerich J. Postgastrectomy syndromes - diagnosis and treatment. Best Pract Res Clin Gastroenterol 2004; 18: 917-933.

45 Dai G, Phalen S, McMurray DN. Nutritional modulation of host responses to mycobacteria. Front Biosci 1998; 3: e110-e122.

46 Cegielski JP, McMurray DN. The relationship between malnutrition and tuberculosis: evidence from studies in humans and experimental animals. Int J Tuberc Lung Dis 2004; 8: 286-298.

47 Wood R, Maartens G, Lombard CJ. Risk factors for developing tuberculosis in HIV-1-infected adults from communities with a low or very high incidence of tuberculosis. J Acquir Immune Defic Syndr 2000; 23: 75-80.

48 Selwyn PA, Hartel D, Lewis VA, et al. A prospective study of the risk of tuberculosis among intravenous drug users with human immunodeficiency virus infection. N Engl J Med 1989; 320: 545-550.

49 Dobler CC, Marks GB. Risk of tuberculosis among contacts in a low-incidence setting. Eur Respir J 2013; 41: 1459-1461.

50 Tubach F, Salmon D, Ravaud P, et al. Risk of tuberculosis is higher with anti-tumor necrosis factor monoclonal antibody therapy than with soluble tumor necrosis factor receptor therapy: The three-year prospective French Research Axed on Tolerance of Biotherapies registry. Arthritis Rheum 2009; 60: 1884-1894.

51 Crawford J, Dale DC, Lyman GH. Chemotherapy-induced neutropenia: risks, consequences, and new directions for its management. Cancer 2004; 100: 228-237.

52 Goldstraw P, Chansky K, Crowley J, et al. The IASLC Lung Cancer Staging Project: Proposals for Revision of the TNM Stage Groupings in the Forthcoming (Eighth) Edition of the TNM Classification for Lung Cancer. $J$ Thorac Oncol 2016; 11: 39-51. 